\title{
The effective s?d exchange interaction: An exact result
}

\section{Raymond Chan \& MiklÓs GulÁcsi}

To cite this article: Raymond Chan \& MiklÓs GulÁcsi (2001) The effective s?d exchange interaction: An exact result, Philosophical Magazine Letters, 81:9, 673-682, DOI: 10.1080/09500830110062780

To link to this article: http://dx.doi.org/10.1080/09500830110062780

册 Published online: 14 Nov 2010.

Submit your article to this journal $\widetilde{ }$

山 Article views: 29

Q View related articles $₫$

4 Citing articles: 10 View citing articles 


\title{
The effective s-d exchange interaction: an exact result
}

\author{
RaYmond Chan and Miklós GuláCsi† \\ Department of Theoretical Physics, Institute of Advanced Studies, \\ The Australian National University, Canberra, Australian Capital Territory \\ 0200, Australia
}

[Received 20 March 2001 and accepted 12 April 2001]

\begin{abstract}
The effective $s-d$ spin interaction is derived exactly for the one-impurity Anderson model via a unitary transformation. The unitary transformation has been calculated up to infinite order, thus an exact transformation was performed in the strict mathematical sense.
\end{abstract}

Schrieffer and Wolff (1966) have established the well known relationship between the Anderson and the Kondo Hamiltonian for more than 30 years, which has revealed the basic magnetic properties of the Anderson-type materials. This relationship is, however, not exact as the canonical transformation involved has only been evaluated up to the first order. A thorough study of this transformation to higher orders is, therefore, bound to shed more light on the novel magnetic characteristics of the Anderson model which are as yet unknown.

In this letter, we carry out this transformation exactly up to infinite order. Higher order terms inevitably generate millions of new terms. The low-energy contributions of these new terms are, however, not all distinct and can be summarized in a form in which each term has a unique set of coefficients. The complexity of the coefficients increases with increasing order of the transformation. After reaching the fifth order, a pattern surprisingly emerges out of the spin interaction coefficients $J_{k}$ which allows us to predict this coefficient to any higher order. This pattern has been verified up to the eleventh order and proven valid to any order by mathematical induction. This is the first time that a canonical transformation of a fully interacting three-dimensional model has been calculated up to infinite order, thus an exact transformation was performed in the strict mathematical sense.

The transformation works in any dimensions and for any number of impurities (even for the periodic Anderson model) with similar results. Hereafter we use the transformation of the original Anderson Hamiltonian containing a single localized orbital $\mathrm{d}$ (or f). The starting Hamiltonian is $H_{0}+H_{V}$, with $H_{0}=\sum_{\mathbf{k}, \sigma} \epsilon(\mathbf{k}) c_{\mathbf{k}, \sigma}^{\dagger} c_{\mathbf{k}, \sigma}+\varepsilon_{\mathrm{d}} \sum_{\sigma} n_{\mathrm{d}, \sigma}+U n_{\mathrm{d}, \uparrow} n_{\mathrm{d}, \downarrow}$ and $H_{V}=\sum_{\mathbf{k}, \sigma}\left(V_{\mathbf{k d}} c_{\mathbf{k}, \sigma}^{\dagger} c_{\mathrm{d}, \sigma}+\mathrm{hc}\right)$, where $\epsilon(\mathbf{k})$ and $\varepsilon_{\mathrm{d}}$ are the one-electron energies of the conduction and localized electrons respectively while the rest of the notation is standard. There is no constraint on the value of $\varepsilon_{\mathrm{d}}$, and consequently the results obtained are also valid for the general asymmetric Anderson model.

$\dagger$ Email: miklos.gulacsi@anu.edu.au

Philosophical Magazine Letters ISSN 0950-0839 print/ISSN 1362-3036 online (C) 2001 Taylor \& Francis Ltd 
The transformed Hamiltonian $\exp (\mathcal{S})\left(H_{0}+H_{V}\right) \exp (-\mathcal{S})$ can be written in the form

$$
H_{0}+\sum_{n=1}^{\infty}[1 / n !-1 /(n+1) !] H_{n},
$$

with

and

$$
\mathcal{S}=\sum_{\mathbf{k}, \sigma}\left(A_{\mathbf{k}}+Z_{\mathbf{k}} c_{\mathrm{d},-\sigma}^{\dagger} c_{\mathrm{d},-\sigma}\right)\left(V_{\mathbf{k d}} c_{\mathbf{k}, \sigma} c_{\mathrm{d}, \sigma}^{\dagger}-\mathrm{hc}\right)
$$

$$
H_{n}=\overbrace{[\mathcal{S},[\mathcal{S},[\mathcal{S}, \ldots[\mathcal{S}}^{n \text { times }}, H_{V}] \cdots]]],
$$

where $A_{\mathbf{k}}=1 /\left[-\epsilon(\mathbf{k})+\varepsilon_{\mathrm{d}}\right], Z_{\mathbf{k}}=1 /\left[-\epsilon(\mathbf{k})+\varepsilon_{\mathrm{d}}+U\right]-1 /\left[-\epsilon(\mathbf{k})+\varepsilon_{\mathrm{d}}\right]$. This satisfies the equation $H_{V}+\left[\mathcal{S}, H_{0}\right]=0$. Note that the transformed Hamiltonian terms $H_{n}$ are proportional to $V^{n+1}$, hence corresponding to the $2 n$ perturbation expansion in $H_{V}$ (Hewson 1993); for example the first transformed Hamiltonian term $H_{1}$ corresponds to the second order perturbation result, the third transformed Hamiltonian term to the sixth-order perturbation result, etc.

The first term of the transformation is the well-known Schrieffer-Wolff (1966) result:

$$
\begin{aligned}
H_{1}= & \sum_{\mathbf{k}, \mathbf{k}^{\prime}, \sigma} V_{\mathbf{k d}} V_{\mathbf{k}^{\prime} \mathrm{d}}\left\{\left[\left(A_{\mathbf{k}}+A_{\mathbf{k}^{\prime}}\right)+\left(Z_{\mathbf{k}}+Z_{\mathbf{k}^{\prime}}\right) n_{\mathrm{d}}\right]\left(n_{\mathrm{d}, \sigma} \delta_{\mathbf{k}, \mathbf{k}^{\prime}}-c_{\mathbf{k}, \sigma}^{\dagger} c_{\mathbf{k}^{\prime}, \sigma}\right)\right. \\
& \left.+\left(Z_{\mathbf{k}}+Z_{\mathbf{k}^{\prime}}\right)\left[c_{\mathbf{k},-\sigma}^{\dagger} c_{\mathbf{k}^{\prime}, \sigma} c_{\mathrm{d}, \sigma}^{\dagger} c_{\mathrm{d},-\sigma}-\frac{1}{2}\left(c_{\mathbf{k},-\sigma}^{\dagger} c_{\mathbf{k}^{\prime}, \sigma}^{\dagger} c_{\mathrm{d},-\sigma}^{\dagger} c_{\mathrm{d}, \sigma}+c_{\mathrm{d},-\sigma}^{\dagger} c_{\mathrm{d},-\sigma} c_{\mathbf{k},-\sigma} c_{\mathbf{k}^{\prime}, \sigma}\right)\right]\right\} .
\end{aligned}
$$

The coefficient of the term $c_{\mathbf{k}, \sigma}^{\dagger} c_{\mathbf{k}^{\prime},-\sigma} c_{\mathrm{d},-\sigma}^{\dagger} c_{\mathrm{d}, \sigma}$ gives the strength $J_{1}$ of the s-d exchange interaction. To calculate the value of $J_{1}$ we keep only the low-energy contributions (Schrieffer and Wolff 1966); hence $\epsilon(\mathbf{k})$ will depend on $|\mathbf{k}|(\equiv k)$ and all the $k$ values are approximately $k_{\mathrm{F}}$. In this way we obtain again the well-known Schrieffer-Wolff result: $J_{1}=2 V^{2} Z$, where $V \equiv V_{k_{\mathrm{F}}}, A \equiv A_{k_{\mathrm{F}}}$ and $Z \equiv Z_{k_{\mathrm{F}}}$. This notation will be used throughout this letter.

Under these conditions, the second-order term becomes

$$
H_{2}=\sum_{\mathbf{k}, \sigma}\left(\tilde{V}_{2}+\tilde{V}_{2}^{c} n_{\mathbf{k}, \sigma}+\tilde{V}_{2}^{d} n_{\mathrm{d}, \sigma}+\tilde{V}_{2}^{c d} n_{\mathbf{k}, \sigma} n_{\mathrm{d}, \sigma}\right)\left(c_{\mathbf{k},-\sigma}^{\dagger} c_{\mathrm{d},-\sigma}+\mathrm{hc}\right),
$$

where $\quad \tilde{V}_{2}=-4 A^{2} V^{3}, \quad \tilde{V}_{2}^{c}=2 Z V^{3}(A-Z), \quad \tilde{V}_{2}^{d}=-2 Z V^{3}(5 A-4 Z) \quad$ and $\tilde{V}_{2}^{c d}=6 Z^{2} V^{3}$. Usually these (and subsequently all even-order) terms are neglected for the reason that they only renormalize the starting Hamiltonian. However, this is not entirely correct. A close inspection of equation (3) reveals that the second-order (and all even-order) terms reflect all possible single-particle virtual processes (and two-particle virtual processes starting from $n \geqslant 3$ ) that can occur between the energy (shells) of the impurity site. The presence of the new terms in equation (3) will have radical consequences for the behaviour of the Kondo exchange coupling constant, which in the third order can be expressed as 


$$
J_{3}=2 V\left[Z\left(\tilde{V}_{2}+\tilde{V}_{2}^{d}\right)-A\left(\tilde{V}_{2}^{c}-\tilde{V}_{2}^{d}\right)\right],
$$

or explicitly $J_{3}=-2^{4} V^{4}\left(2 A^{2} Z+2 A Z^{2}+Z^{3}\right)$.

The contributions of $H_{1}+H_{3}$ are plotted in figure 1 which shows that the Kondo exchange has reversed sign. In perturbation theory, the $J_{3}$ term corresponds to a sixth-order process (Hewson 1993) of two exchanged electrons. This phenomenon is the Friedel (i.e. Ruderman-Kittel) oscillations, where the polarization periodically reverses as one proceed to higher orders. Similar oscillations can also be observed in the renormalization group approach (Hewson 1993) in terms of energy shells.

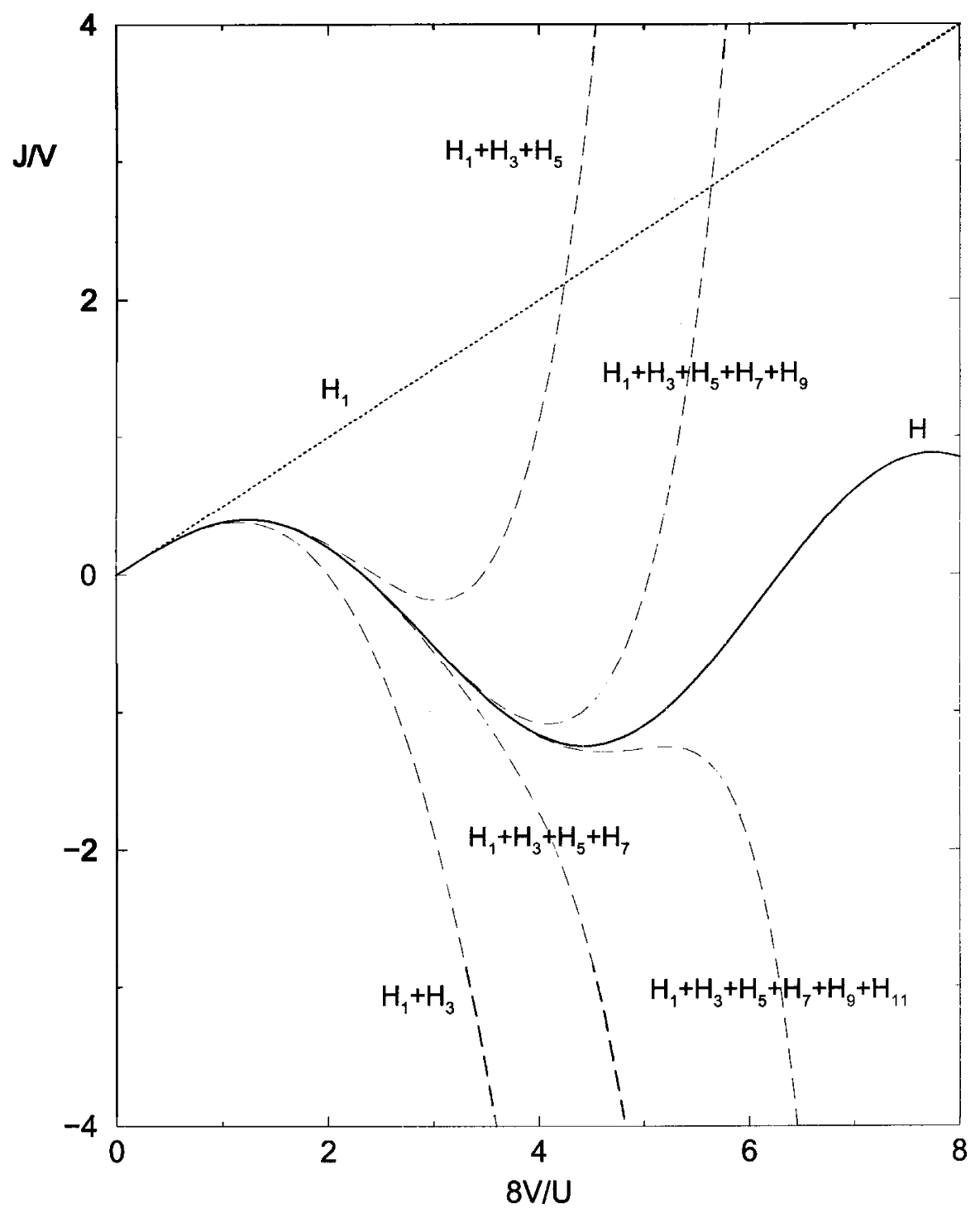

Figure 1. The Schrieffer-Wolff result $\mathrm{H}_{1}(\ldots \ldots)$ is compared with the first- third-, fifth-, seventh-, ninth- and eleventh-order contributions (- - $)$. $\mathrm{H}(-)$ is the exact result, where all the infinite-order contributions were summed. For simplicity all the curves were plotted for the symmetric case $\varepsilon_{\mathrm{d}}=-U / 2$ relative to $\epsilon\left(k_{\mathrm{F}}\right)=0$. 
This sign oscillation is important for our analysis, as this will guarantee that the $J_{n}$ series is convergent. In general terms, these oscillations are due to the multiplescattering processes between the excitated levels. The sign oscillation emerging in the $U \rightarrow 0$ limit arises because the triplet and singlet excited states become degenerate.

This can be seen easily if we calculate the energy levels of the few-electron case. Since a site can accommodate at most four electrons, the eigenstates of the electrons can be easily calculated. These eigenstates will separate into at least four different states, with one, two, three and four electrons. With four electrons, no hybridization will be allowed as no vacancies are provided for hopping. Therefore, only three cases will be of interest to us.

The one electron case contains the combination of a single $\mathrm{d}$ and $\mathrm{c}$ electrons with spin $\sigma$. There are two different energy states associated with this combination for singlet and triplet:

$$
\sigma_{\mp}=\left[c_{\sigma}^{\dagger} \sin \theta \mp d_{\sigma}^{\dagger} \cos \theta\right]|0\rangle .
$$

where $|0\rangle$ is the empty ionic state, and $\sin \theta$ and $\cos \theta$ take care of the orthonormality condition. The corresponding energies

$$
E_{\sigma \mp}=\frac{\varepsilon_{\mathrm{d}} \mp\left(\varepsilon_{\mathrm{d}}^{2}+4 V^{2}\right)^{1 / 2}}{2},
$$

split into two eigenstate with eigenvalues independent of $U$ (figure 2).

The singlet state of two electrons can be written as the combination of the product of two electrons, in such a way that a spin flip of the whole state will attract a minus sign to it. In terms of electron operators, it can be written as

$$
s=\left[\alpha c_{\uparrow}^{\dagger} c_{\downarrow}^{\dagger}+\beta f_{\uparrow}^{\dagger} f_{\downarrow}^{\dagger}+\gamma\left(f_{\downarrow}^{\dagger} c_{\uparrow}^{\dagger}-f_{\uparrow}^{\dagger} c_{\downarrow}^{\dagger}\right)\right]|\mathrm{i} 0\rangle,
$$

where $\alpha, \beta$ and $\gamma$ are normalized $\left(\alpha^{2}+\beta^{2}+\gamma^{2}=1\right)$ ratios of the three different electron configurations. To equation (7) always corresponds three real and distinct solutions, given the positive definite values of the energy parameters $\varepsilon_{\mathrm{d}}, U$ and $V$ :

$$
E_{\mathrm{s}}=-\frac{2 V}{\alpha}, \quad E_{\mathrm{s}}=2 \varepsilon_{\mathrm{d}}+U-\frac{2 V \gamma}{\beta}, \quad E_{\mathrm{s}}=\varepsilon_{\mathrm{d}}-\frac{V(\alpha+\beta)}{\gamma},
$$

while the coefficients $\alpha, \beta$ and $\gamma$ are given by

$$
\alpha=-\frac{2 V \gamma}{E_{\mathrm{s}}}, \quad \beta=\frac{2 V \gamma}{2 \varepsilon_{\mathrm{d}}+U-E_{\mathrm{s}}}, \quad \gamma=\frac{\alpha+\beta}{\varepsilon_{\mathrm{d}}-E_{\mathrm{s}}} V .
$$

Substituting $\alpha$ and $\beta$ from equation (9) into equation (8) and eliminating $\gamma$ give a cubic equation for $E_{\mathrm{s}}$ with fairly complicated solutions. For $U=0$ the solutions are:

$$
E_{\mathrm{S}_{1}}=\varepsilon_{\mathrm{d}}-\left(\varepsilon_{\mathrm{d}}^{2}+4 V^{2}\right)^{1 / 2}, \quad E_{\mathrm{s}_{2}}=\varepsilon_{\mathrm{d}}, \quad E_{\mathrm{s}_{3}}=\varepsilon_{\mathrm{d}}+\left(\varepsilon_{\mathrm{d}}^{2}+4 V^{2}\right)^{1 / 2} .
$$

When $U \rightarrow \infty, E_{\mathrm{s}_{3}}$ goes to $+\infty$, and the remaining two eigenvalues are

$$
E_{\mathrm{s}_{1 / 2}}=\frac{\varepsilon_{\mathrm{d}} \mp\left(\varepsilon_{\mathrm{d}}^{2}+8 V^{2}\right)^{1 / 2}}{2} \text {. }
$$

Interestingly, the triplet eigenstate

$$
t=\frac{1}{2^{1 / 2}}\left(f_{\downarrow}^{\dagger} c_{\uparrow}^{\dagger}+f_{\uparrow}^{\dagger} c_{\downarrow}^{\dagger}\right)|\mathrm{i} 0\rangle
$$




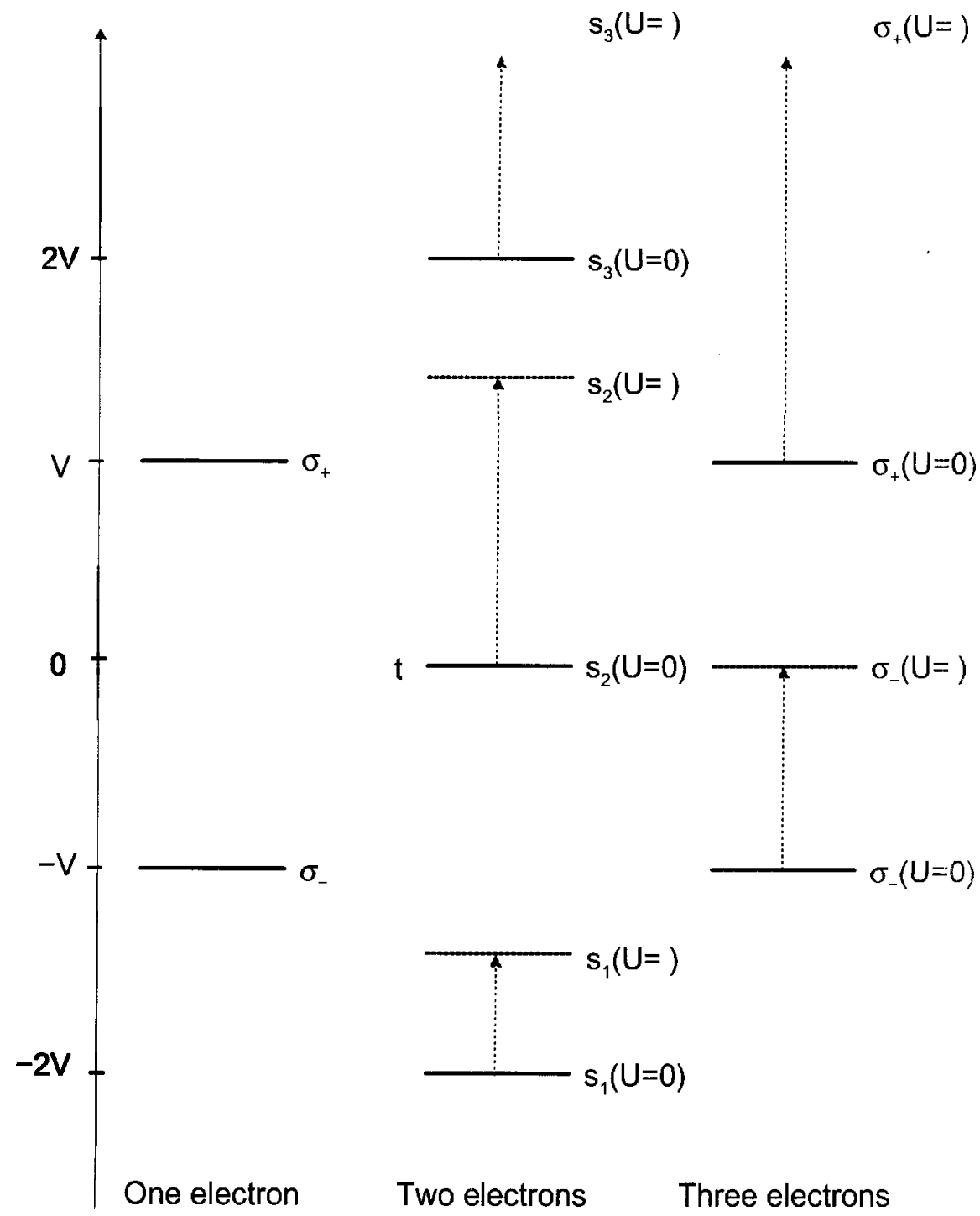

Figure 2. Energy levels of a one-, two- and three-electron limit. For simplicity we have considered $\varepsilon_{\mathrm{d}}=0$.

yields only one eigenvalue $\varepsilon_{\mathrm{d}}$, independent of $V$ and $U$. Thus, for $U=0$ the energy level of one of the singlet states is degenerate with the triplet state (see figure 2).

The singlet and triplet eigenstates of three electrons can be written, similarly to the one electron states, as

$$
\sigma_{\mp}=\left[f_{\sigma}^{\dagger} c_{\uparrow}^{\dagger} c_{\downarrow}^{\dagger} \sin \theta \mp c_{\sigma}^{\dagger} f_{\uparrow}^{\dagger} f_{\downarrow}^{\dagger} \cos \theta\right]|0\rangle,
$$

with corresponding energies

$$
E_{\sigma \mp}=\frac{3}{2} \varepsilon_{\mathrm{d}}+\frac{1}{2} U \mp \frac{1}{2}\left[\left(\varepsilon_{\mathrm{d}}+U\right)^{2}+4 V^{2}\right]^{1 / 2} .
$$


A close inspection of figure 2 shows that the behaviour of three electrons is very similar to that in the one-electron case.

The energy difference of the singlet and triplet states is about $2 \mathrm{~V}$ with a singleatom model. In the Anderson model when the number of free electrons is not limited to two, this energy difference will be reduced and the two states could coexist and localize in different regions.

As we continued further to the higher-order terms, the complexity of the result increased dramatically and soon became incomprehensible. Hence to deal with this enormous task of algebraic manipulation, a symbolic program has been written to perform the laborious fermionic commutations and the fermionic algebra.

As mentioned earlier, the usual simplification scheme (Schrieffer and Wolff 1966, Hewson 1993) used after each step is to keep only the low-energy contributions, that is the terms for which the $k$ values are approximately $k_{\mathrm{F}}$. This reduces the complexity to a manageable level. In this way we have calculated the transformed Hamiltonian up to the eleventh order, $H_{11}$. However, even in this case the total number of terms which are generated in each order remains enormous: 32 for $n=2 ; 236$ for $n=3$; 1152 for $n=4 ; 10008$ for $n=5 ; 48320$ for $n=6 ; 420080$ for $n=7 ; 1994496$ for $n=8 ; 17157984$ for $n=9 ; 80991488$ for $n=10 ; 692245952$ for $n=11$. Each odd order $(n \geqslant 3)$ contributes $2 \times 2^{n+(n-3) / 2} \times 5^{(n-1) / 2}$ terms to the Kondo exchange.

We observed from these results that the even-order terms $H_{2}, H_{4}, H_{6}, \ldots$ of the canonical transformation all contain terms or combinations of terms similar to equation (3). On the other hand, the odd-order terms $H_{1}, H_{3}, H_{5}, \ldots$ of the transformation, always contain a Kondo exchange term with coefficient given by equation (4), with the left-hand side being of the $(n+1)$ th order, and the right-hand side the $n$th order of the transformation. The calculated $J_{n}$ values for $n \geqslant 5$ are

$$
\begin{aligned}
J_{5}= & 2^{7} V^{6}\left(4 A^{4} Z+8 A^{3} Z^{2}+8 A^{2} Z^{3}+4 A Z^{4}+Z^{5}\right), \\
J_{7}= & -2^{10} V^{8}\left(8 A^{6} Z+24 A^{5} Z^{2}+36 A^{4} Z^{3}+32 A^{3} Z^{4}\right. \\
& \left.+18 A^{2} Z^{5}+6 A Z^{6}+Z^{7}\right), \\
J_{9}= & 2^{13} V^{10}\left(16 A^{8} Z+64 A^{7} Z^{2}+128 A^{6} Z^{3}+160 A^{5} Z^{4}\right. \\
& \left.+136 A^{4} Z^{5}+80 A^{3} Z^{6}+32 A^{2} Z^{7}+8 A Z^{8}+Z^{9}\right), \\
J_{11}= & -2^{16} V^{12}\left(32 A^{10} Z+160 A^{9} Z^{2}+400 A^{8} Z^{3}\right. \\
& +640 A^{7} Z^{4}+720 A^{6} Z^{5}+592 A^{5} Z^{6}+160 A^{3} Z^{8} \\
& \left.+360 A^{4} Z^{7}+50 A^{2} Z^{9}+10 A Z^{10}+Z^{11}\right) .
\end{aligned}
$$

The results up to eleventh order are presented in figure 1. All these calculated $J_{n}$ $(1 \leqslant n \leqslant 11)$ coefficients appear to have a pattern over different orders:

$$
(-1)^{n-1} 2^{3(n-1) / 2}\left(\frac{1}{n !}-\frac{1}{(n+1) !}\right) Z V^{n+1}\left[(Z+A)^{2}+A^{2}\right]^{(n-1) / 2} .
$$

In a final step, equation (16) can be proven to be valid to any order by induction; assuming that equation (16) is true for any (odd) $n$, it is straightforward to carry out the commutation of equation (1) for $n+1$ and then for $n+2$. The $(n+2)$ th order will again contain equation (16), with $n$ replaced by $n+2$. After each step we keep only the low-energy contributions, that is terms for which the $k$ values are approxi- 
mately $k_{\mathrm{F}}$. The proof is not difficult but extremely tedious, and hence will be presented in a later publication.

Summing the coefficients from equation (16) to infinity we obtain a remarkably simple result:

$$
J=2 Z V^{2}\left(\frac{\sin \beta}{\beta}+\frac{\cos \beta-1}{\beta^{2}}\right),
$$

where

$$
\beta=\left\{2^{3} V^{2}\left[(Z+A)^{2}+A^{2}\right]\right\}^{1 / 2} .
$$

For the symmetric case, where $\varepsilon_{\mathrm{d}}=-U / 2, J$ becomes

$$
J_{\text {sym }}=V \sin (2 V Z)+\frac{1}{2 Z}[\cos (2 V Z)-1] .
$$

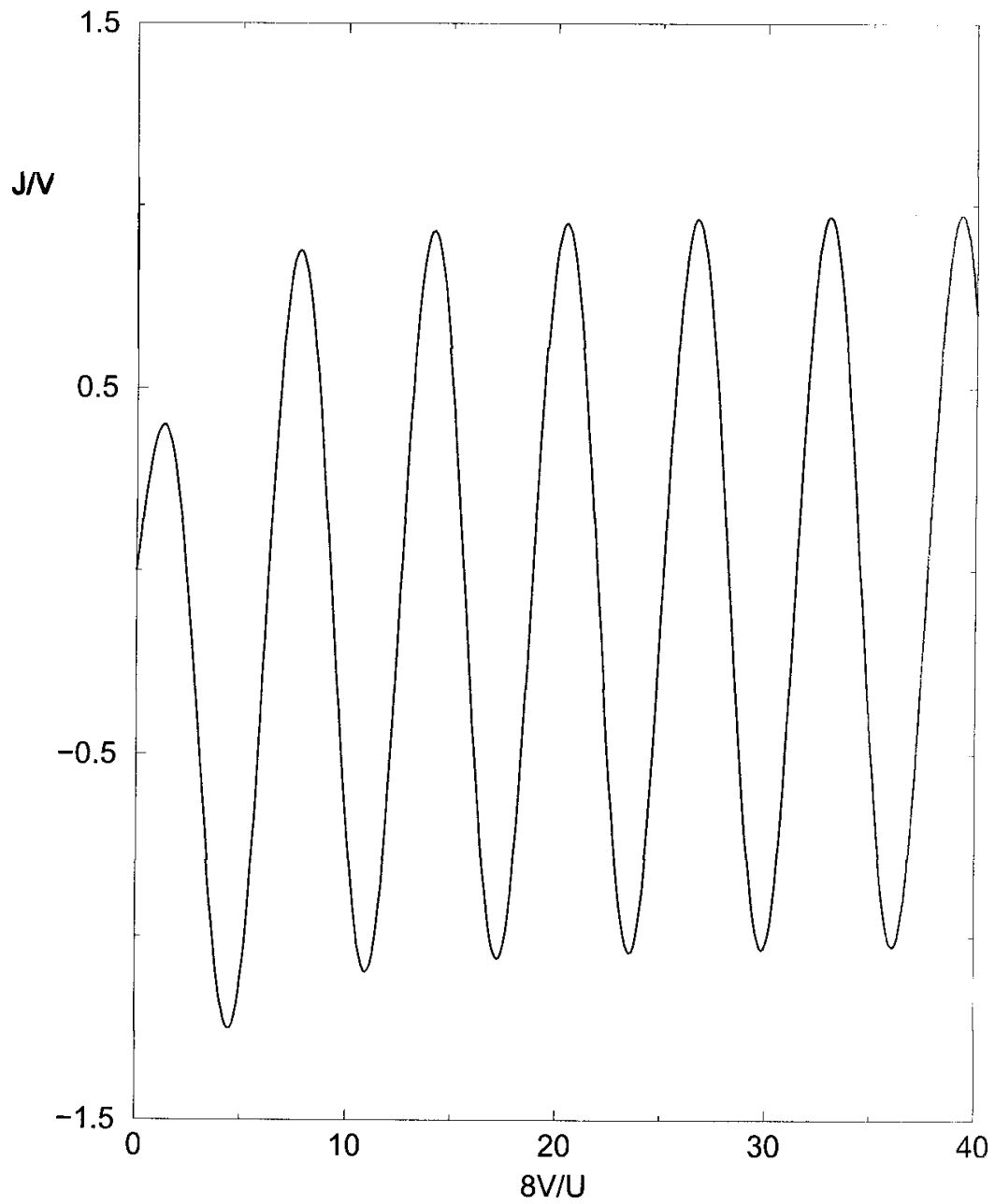

Figure 3. The exact Kondo exchange interaction (17) is plotted as a function of $8 \mathrm{~V} / U$ for the symmetric case, $\varepsilon_{\mathrm{d}}=-U / 2$. For simplicity we took $\epsilon\left(k_{\mathrm{F}}\right)=0$. 


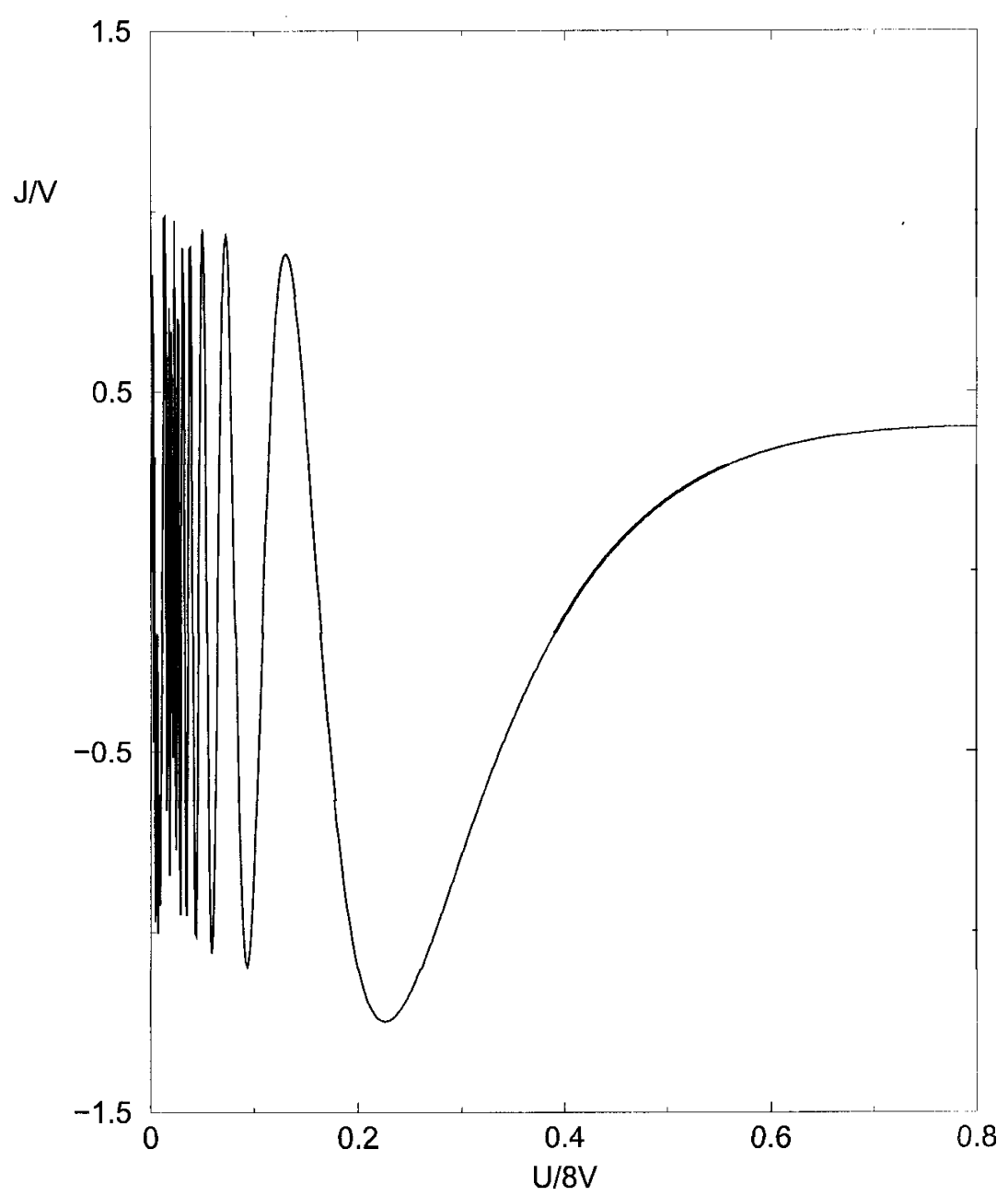

Figure 4. The same as in figure 3 , but as a function of $U / 8 \mathrm{~V}$. This way of plotting equation (17) shows clearly the two different scaling limits: local moment regime (Kondo limit) for $U \gg V^{2}$ and the mixed valence regime, $\varepsilon_{\mathrm{d}} \approx \epsilon\left(k_{\mathrm{F}}\right)$.

For simplicity we consider $\epsilon\left(k_{\mathrm{F}}\right)=0$ hereafter. For this case the results for the symmetric Anderson model are plotted in figure 3 as a function of $8 \mathrm{~V} / \mathrm{U}$ and in figure 4 as a function $U / 8 \mathrm{~V}$.

The two fixed points of the symmetric Anderson model can be seen clearly in figures 3 and 4 . For $U \gg V^{2}$, equation (19) reduces to

$$
J_{\text {sym }} \approx \frac{U}{8}\left[\cos \left(\frac{8 V}{U}\right)-1\right] .
$$

In the limit $U \rightarrow \infty$, this equation reduces to the well-known Schrieffer-Wolff result of the local moment or Kondo regime. This regime has been extensively studied in the past. However, this is not the case for smaller values of $U$.

As $U$ decreases, $V^{2} \gg U$ and $\varepsilon_{\mathrm{d}}$ approaches $\epsilon\left(k_{\mathrm{F}}\right)$, we cross over the mixedvalence regime. The two bands $\mathrm{s}$ and $\mathrm{d}$ (or f) overlap, which causes the virtual excitations of the impurity energy levels (shells) and the s-d multiple-scattering 
processes to dominate. These processes cause most of the perturbative approaches to fail (Hewson 1993). However, our transformation is still convergent. For $U \rightarrow 0$ equation (19) reduces to

$$
J_{\mathrm{sym}} \approx V \sin \left(\frac{8 V}{U}\right),
$$

As mentioned earlier, the strong oscillations which appear at very small values of $U$ (clearly seen in figure 4) are due to the multiple-scattering processes between singlet and triplet states.

The zeros of equation (19) can be determined easily to be the solutions of $\sin (4 V / U)=0$ or $8 V / U=\tan (4 V / U)$. These points represent the crossover between antiferromagnetic and ferromagnetic $\mathrm{s}-\mathrm{d}$ exchange interaction. The first of the zeros, corresponding to the largest $U$ value, $8 V^{2} / U \approx 2.33$, was first looked at by Clogston and Anderson (1961). However, from equation (19) it can be seen that there is a whole range of $V^{2} / U$ when the ferromagnetic polarization of s electrons caused by admixture with localized inner-shell levels is exactly cancelled by the antiferromagnetic polarization effect.

These fluctuations are not present in the asymmetric Anderson model, as shown in figure 5 where equation (15) is plotted for different values of $\varepsilon_{\mathrm{d}}$. When there is no

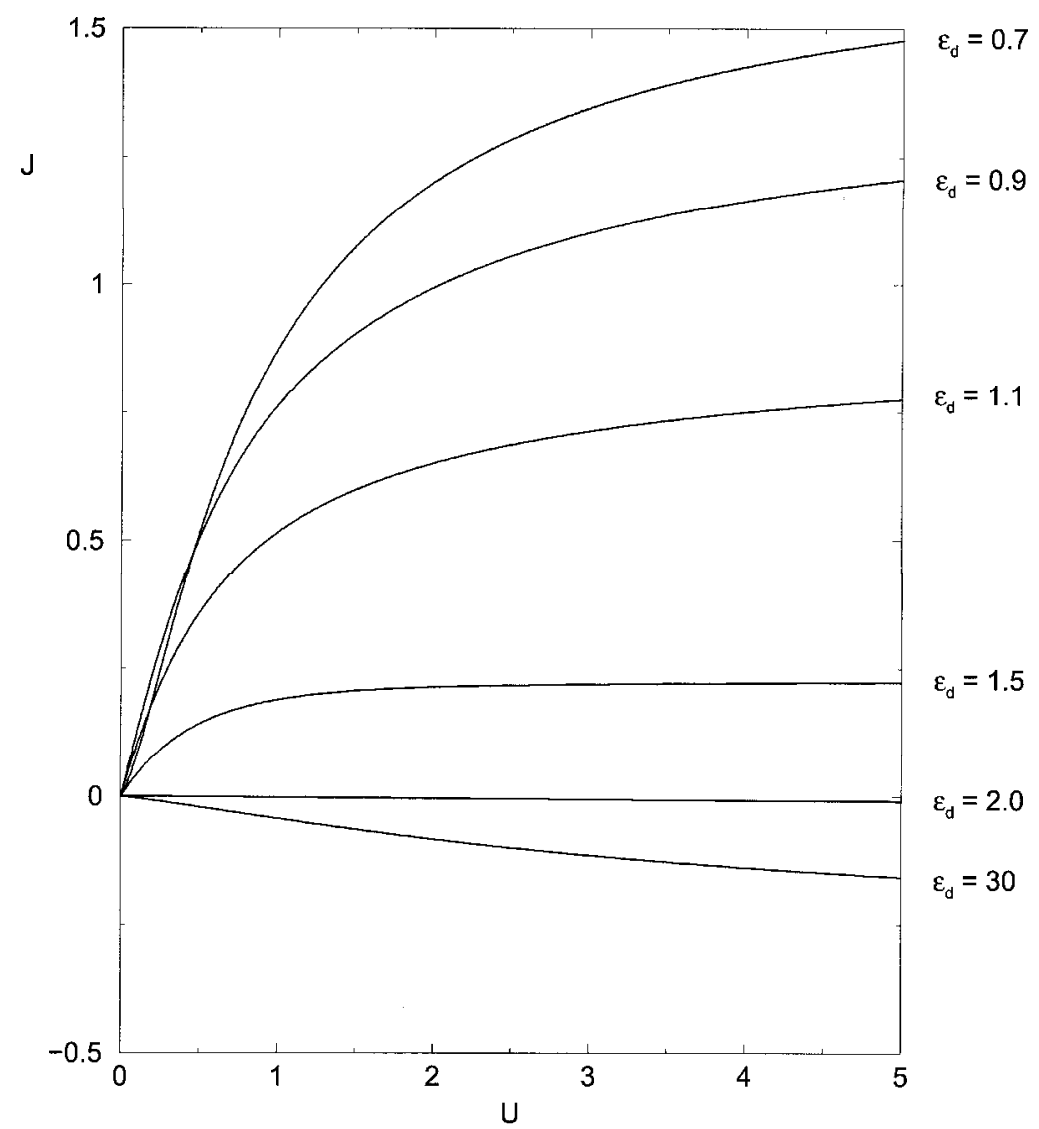

Figure 5. Several asymmetric cases are presented for the simplified case of $V=1$ and $\epsilon\left(k_{\mathrm{F}}\right)=0$. 
s-d mixing, multiple-scattering processes are absent and the oscillations in $J$ disappear completely.

In conclusion, we have performed an exact canonical transformation of the Anderson model. The transformation was carried out up to infinite order and all terms are summed. This allowed us to calculate rigorously the strength of the s-d exchange Hamiltonian (retarded self-energy) for any value of $U$ and/or $V$.

\section{ACKNOWLEDGEMENTS}

Preliminary results were presented and/or discussed during the International Conference on Concepts in Electron Correlation, Hvar, 27-29 September 1999. On of the authors (M.G.) acknowledges fruitful discussion with P. W. Anderson, A. Bussmann-Holder, B. Coqblin, J. E. Gubernatis, Zs. Gulacsi, A. C. Hewson, J. Hirsch, A. M. Oles, P. S. Riseborough, A. Rosengren, J. L. Smith and V. Zlatic.

\section{REFERENCES}

Clogston, A. M., and Anderson, P. W., 1961, Bull. Am. phys. Soc., 6, 124.

Hewson, A. C., 1993, The Kondo Problem to Heavy Fermions (Cambridge University Press). SChriefFer, J. R., and WolfF, P. A., 1966, Phys. Rev., 149, 2. 